\title{
Fenotipo grave en dos medio hermanas con síndrome de Adams Oliver
}

\author{
Severe phenotype in two half-sibs with Adams Oliver syndrome
}

\author{
Dra. Rosalba Sevilla-Montoya ${ }^{a}$, Dr. Braulio Ríos-Flores ${ }^{b}$ Dra. Elsa Moreno-Verduzcoc, \\ Biol. Mauricio Dominguez-Castro ${ }^{a}$, Dr. Carlos I. Rivera-Pedroza y Dra. Mónica Aguinaga-Ríos ${ }^{a}$
}

\section{RESUMEN}

El síndrome de Adams Oliver (AOS) es una entidad heterogénea con defecto transverso terminal de extremidades (TTLD) y aplasia cutis congénita (ACC) con un amplio espectro fenotípico. Se han descrito diferentes modos de herencia en esta enfermedad; los defectos más graves se han asociado a un patrón autosómico recesivo (AR).

Objetivo. presentar a una familia con dos medio hermanas con un fenotipo grave de Adams Oliver, con una madre sana. Reporte del caso: una mujer de 27 años de edad fue referida al Departamento de Genética. Su hija anterior presentó acránea, anillos de constricción y defectos transversos terminales de extremidades. Su hija actual presentaba encefalocele occipital, defecto amplio en huesos del cráneo, aplasia cutis congénita, defecto terminal transverso de extremidades y labio y paladar hendido bilateral. Sugerimos que algunos casos con fenotipo grave del síndrome de Adams Oliver pueden deberse a herencia autosómico dominante con penetrancia incompleta o a la presencia de mosaicismo gonadal.

Palabras clave: Adams Oliver, aplasia cutis, defecto terminal transverso de extremidades.

\begin{abstract}
Adams Oliver syndrome (AOS) is a highly variable entity with terminal transverse limb defects (TTLD) and aplasia cutis congenita (ACC) with a wide phenotypic spectrum. Several inheritance models have been observed; the most severe phenotype has been related to an autosomal recessive (AR) pattern of inheritance.

Objective. To present a family with two half siblings with a severe phenotype of Adams Oliver syndrome in which the mother was healthy. Case report: A 27 year-old woman was referred to the Genetics Department. Her previous girl presented acrania, constriction rings and terminal transverse limb defects. The present girl had occipital encephalocele, large scalp defects, aplasia cutis congenita, terminal transverse limb defects and bilateral cleft lip and palate. Autosomal dominant
\end{abstract}

a. Departamento de Genética y Genómica del Instituto Nacional de Perinatología.

b. Departamento de Neurología del Instituto Nacional de Perinatología.

c. Departamento de Patología del Instituto Nacional de Perinatología.

México, DF, México.

Correspondencia:

Dra. Mónica Aguinaga-Ríos: aguinagamonica09@gmail.com.

Conflicto de intereses: ninguno que declarar.

Recibido: 28-10-2013

Aceptado: 10-2-2014 inheritance with reduced penetrance or gonadal mosaicism has to be considered in Adams Oliver syndrome with severe intracranial anomalies.

Key words: Adams Oliver, aplasia cutis, terminal transverse limb defect.

http:/ /dx.doi.org/10.5546/aap.2014.e108

\section{INTRODUCCIÓN}

En 1945, Adams y Oliver ${ }^{1}$ fueron los primeros en describir la asociación de aplasia cutis congénita (ACC) con defectos terminales transversos de extremidades (TTLD). Desde entonces, una amplia gama fenotípica ha sido descrita en estos pacientes.

La aplasia cutis congénita se localiza principalmente en el vértex del cráneo y puede asociarse a diferentes grados de daño al periostio, al hueso y a la duramadre. ${ }^{1}$ Algunos reportes describen la presencia de encefalocele ${ }^{2}$ y de acránea ${ }^{3}$ como parte de una presentación grave de este síndrome.

Fryns $^{4}$ fue el primero en describir una probable asociación entre el síndrome de Adams Oliver (AOS) y anomalías intracraneales estructurales. Se ha propuesto que hasta el $32 \%$ de los pacientes con un modelo de herencia autosómico recesivo presentan un fenotipo neurológico grave con microcefalia, retraso mental y anomalías intracraneales. ${ }^{5}$ Sin embargo, estas anomalías también se han visto en algunos casos con un aparente patrón autosómico dominante., 2,6

El objetivo de este artículo es presentar dos medio hermanas con una forma grave del síndrome de Adams Oliver además del hallazgo de una banda amniótica adherida a la placenta, enumerar las etiologías propuestas de este síndrome y sus modelos de herencia.

\section{CASO CLÍNICO}

Una mujer de 27 años, G5, C2, A2, fue referida al Departamento de Genética a las 16 semanas de gestación con un ultrasonido con reporte de feto con hidrocefalia. Dentro de sus antecedentes, encontramos dos pérdidas de embarazos espontáneas del primer trimestre con su pareja 
anterior; un varón nacido aparentemente sano, que presentó muerte sin causa aparente al año y ocho meses de edad; y un feto muerto de sexo femenino de su segunda pareja. Esa niña mostraba acránea y anillos de constricción con defecto transverso terminal en el $4^{\circ}$ y $5^{\circ}$ dedo de la mano izquierda. En esa paciente, se realizó el diagnóstico clínico de secuencia de bandas amnióticas. La necropsia reportó meroacránea, amputación del quinto dedo de mano izquierda, exoftalmos parcial del ojo derecho y completo del ojo izquierdo. Nuestra probando es la hija de la tercera pareja de esta paciente, un varón sano de 26 años con dos hijos sanos de otra pareja. Ambos padres eran completamente sanos, con estudio cromosómico normal. No existía historia materna de abuso de sustancias o infecciones durante el embarazo.

El ultrasonido prenatal estructural a las 25 semanas reportó macrocráneo con ventriculomegalia asimétrica, encefalocele occipital y labio y paladar hendido bilateral. El cariotipo prenatal resultó normal 46, XX. El nacimiento se dio a las 41 semanas de gestación; con un peso de $4570 \mathrm{~g}$ (>percentilo 97), talla de $53 \mathrm{~cm}$ (en el percentilo 90) y una circunferencia cefálica de $51 \mathrm{~cm}$ (>percentilo 99). Se observó un aparente encefalocele, con aplasia cutis en el vértex; las manos mostraban una ausencia de la falange distal del $4^{\circ}$ dedo derecho e hipoplasia de la falange distal y la uña del $3^{\text {er }}$ dedo derecho, con un aparente anillo de constricción (Figura 1). A nivel facial, encontramos cejas escasas, hipoplasia y coloboma del párpado derecho con ausencia del músculo tarsal y orbicular, y una úlcera corneal ipsilateral; hipoplasia medio facial con labio y paladar hendido. En los pies, se observó pie equino varo izquierdo y pie derecho normal. En la tomografía, se detectó plagiocefalia con ausencia de huesos parietales y de tejido cerebral medio, corteza cerebral displásica con desplazamiento anterior, un grave defecto medial óseo en el área frontal y occipital, y una dilatación quística en la fosa posterior con un encefalocele occipital (Figura 2).

El ecocardiograma mostró un ducto arterioso permeable y el ultrasonido renal fue normal.

A la exploración dirigida de la placenta se reportó como hipertrófica (730 g); no se encontraron bandas amnióticas libres; sin embargo, se observó la presencia de una banda fibrosa de $9 \times 1,2 \mathrm{~cm}$ adherida en uno de sus bordes (Figura 3).

Basados en la presencia de aplasia cutis congénita y defecto transverso terminal de
FIGURA 1. Recién nacida, en la que se observa la aplasia cutis congénita en el vértex (a), defecto terminal transverso de extremidad con ausencia de la falange distal del $4^{\text {to }}$ dedo derecho (b) y la hipoplasia de la falange distal y uña del $3^{\text {er }}$ dedo derecho con aparente anillo de constricción (c)

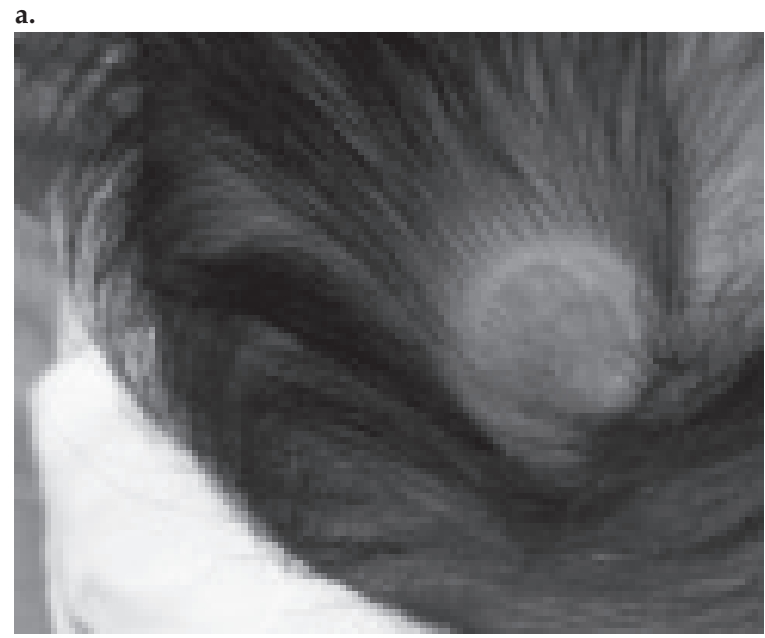

b.

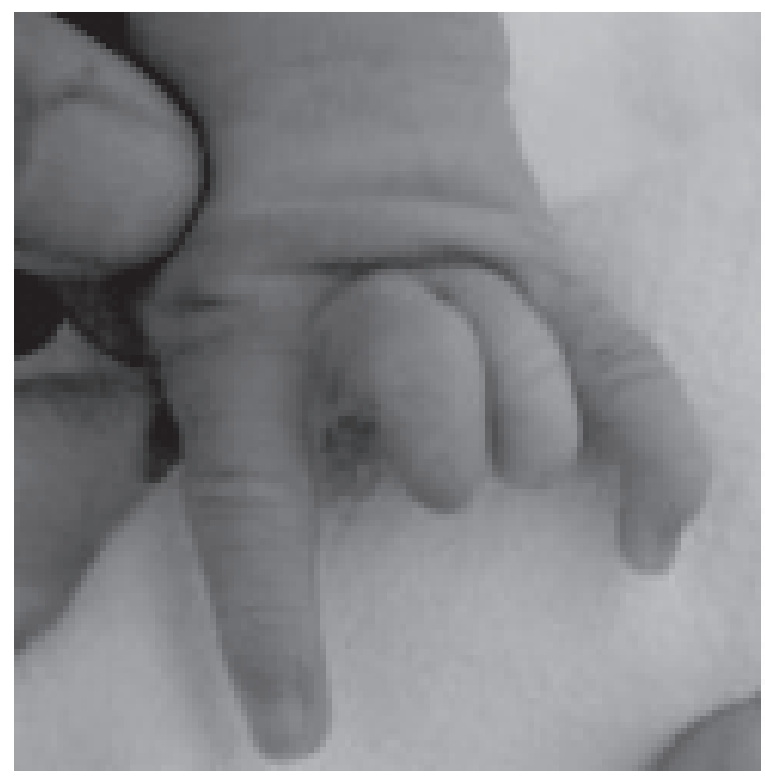

c.

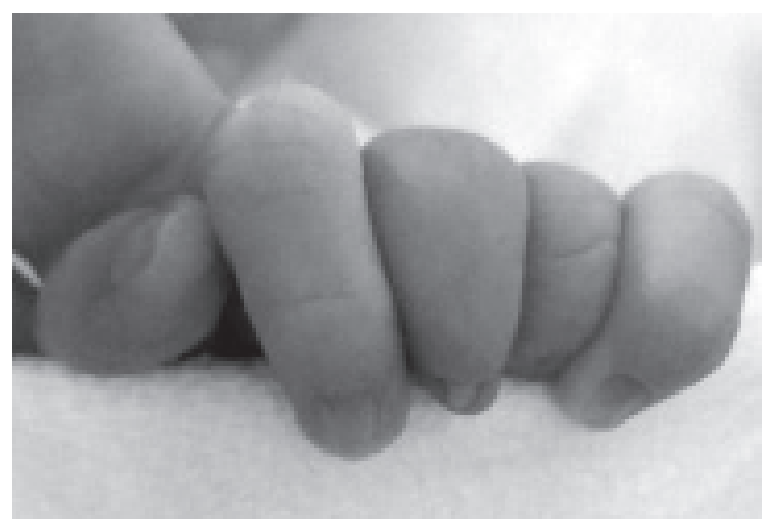


extremidades, se llegó al diagnóstico de síndrome de Adams Oliver. La madre no mostraba defectos craneales ni en las extremidades. Sus estudios radiológicos fueron normales.

\section{COMENTARIO}

Reportamos dos medio hermanas con una forma grave del síndrome de Adams Oliver, en la que la madre no mostraba datos clínicos de esta entidad. La historia clínica sugiere un patrón de herencia autosómico dominante sin poder descartar un mosaicismo gonadal.

Savarirayan y cols., ${ }^{6}$ fueron los primeros en sugerir un mosaicismo gonadal materno en una familia con dos hijos afectados de una madre con hipoplasia ungueal del $5^{\circ}$ dedo. El estudio describe un paciente con displasia cortical grave y anillos de constricción en ambos afectados. El paciente reportado en nuestro estudio presentó defectos intracraneales graves caracterizados por displasia cortical en diversas regiones cerebrales. Esta anomalía representa trastornos en la migración y diferenciación celular en etapas tempranas del embarazo. Nuestro caso apoya la presencia de anomalías intracraneales graves en casos con patrón de herencia autosómico dominante. Aunque la presencia de varios hijos afectados de padres sanos ha sido previamente descrita por Southgate ${ }^{7}$ en dos familias con herencia autosómico dominante, vale la pena destacar que, en este caso, la madre sana tuvo hijos gravemente afectados con dos parejas diferentes.

Hoyme y cols., ${ }^{8}$ describieron la hipótesis de un accidente vascular trombótico en el útero como causa de interrupción del flujo sanguíneo hacia las estructuras en desarrollo.

FIGURA 2. Imágenes neonatales de tomografía computada, que muestran asimetría facial, labio hendido bilateral, amplio defecto craneal (DC), que involucra el frontal, parietales y occipital. El corte axial muestra plagiocefalia. Con una flecha, se indica el desplazamiento anterior de la corteza cerebral, encefalocele occipital (E) y parte del ventrículo lateral izquierdo (VI)
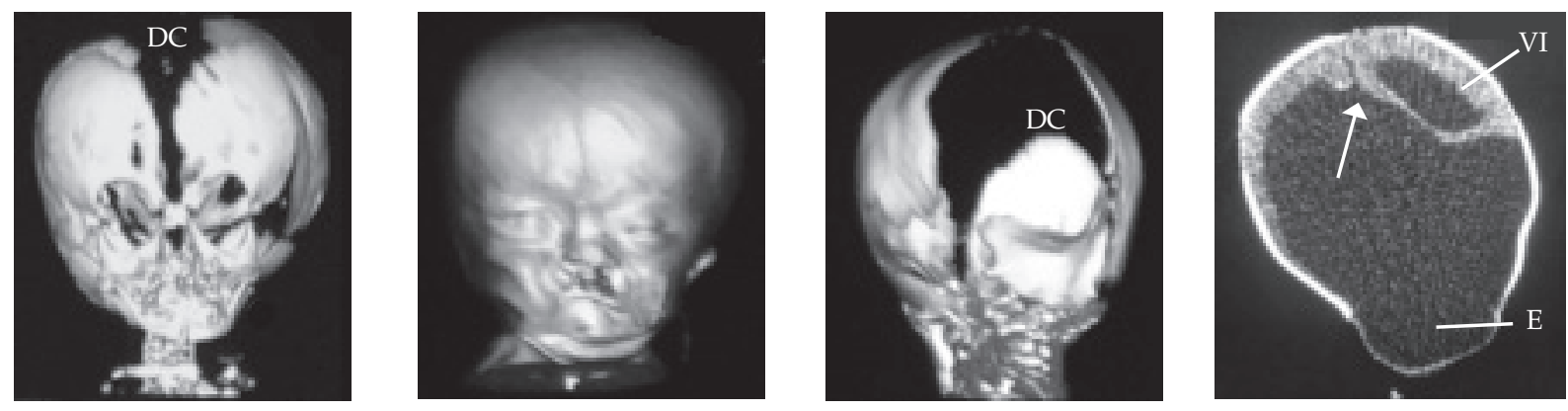

Figura 3. Placenta del propositus, en la que se indica, con una flecha, la presencia de una banda adherida.

a.

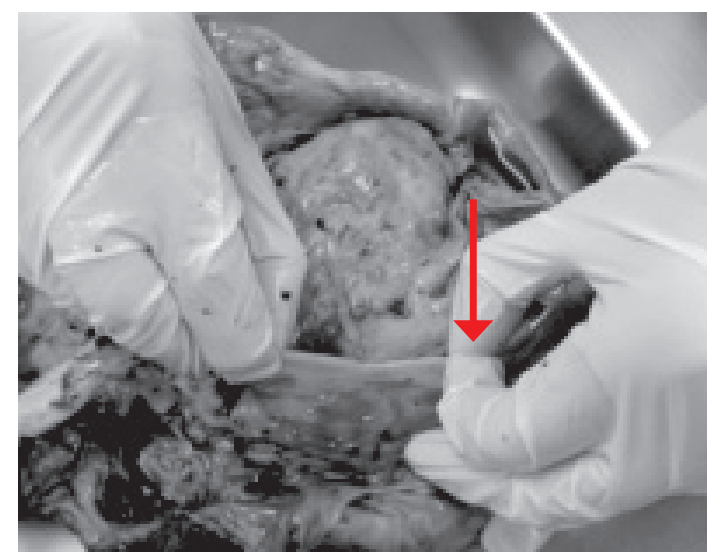

b.

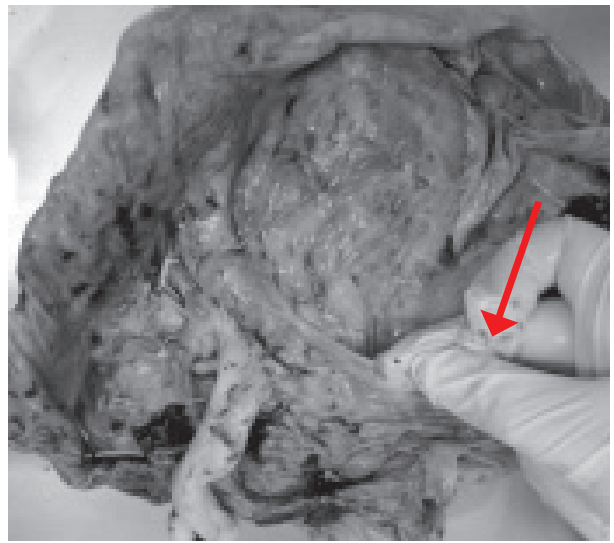


La principal etiología y patogénesis de este síndrome continúa siendo controversial. El análisis molecular en pacientes afectados por este padecimiento revela mutaciones en dos genes, ARHGAP31 y RBPJ, que regulan a miembros de la familia Rho GTPasa y se han relacionado con el modelo de herencia autosómico dominante. ARHGAP31 está involucrado en la regulación de la proliferación y migración celular, especialmente en etapas tempranas del desarrollo, y las mutaciones que causan una activación constitutiva de este gen resultan en una pérdida de Cdc42 activo y originan alteraciones en la actina del citoesqueleto, que se han encontrado en casos autosómico dominantes y expresividad variable. ${ }^{7}$ Esto sugiere que la presencia de alteraciones mesenquimatosas, como la formación de bandas y el daño temprano en estructuras fetales, pueden tener una interacción más estrecha de lo conocido hasta el momento. Hassed estudió la vía de señalización Notch mediada por $R B P J$ en dos pacientes no relacionados y encontró dos mutaciones en este regulador transcripcional importante para la proliferación celular del mesénquima y la formación esquelética, ${ }^{9}$ así como para la formación de epidermis, desarrollo folicular capilar ${ }^{10}$ y estructuras vasculares. ${ }^{11}$

En los casos con modelo de herencia autosómico recesivo, Shasheen y cols. han detectado mutaciones en DOCK $6^{12}$ y recientemente en el gen EOGT, que tiene actividad de $\mathrm{N}$-acetilglucosamiltransferasa y realiza la glicosilación de dominios de proteínas extracelulares no relacionadas con el citoesqueleto, lo cual no se había asociado anteriormente a esta enfermedad y que puede explicar la amplia heterogeneidad de los casos recesivos. ${ }^{13}$

Nuestra paciente tenía el antecedente de un hijo muerto con acránea y el presente caso con encefalocele. Estas anomalías reflejan el grave defecto de osificación craneana sugeridas previamente por Kuster et al. ${ }^{2}$ y reportadas hasta en el $27 \%$ por Farrell. ${ }^{14}$ En estos casos, el diagnóstico clínico es complicado ya que no siempre puede observarse la aplasia cutis. Chitayat et al. ${ }^{3}$ fueron los primeros en describir un paciente con acránea, TTLD y defecto cutáneo. Ellos concluyeron que este fenotipo correspondía a un caso grave de AOS y sugirieron la búsqueda intencional de TTLD en pacientes con anencefalia. Los estudios de imagen de nuestro propósito muestran un amplio defecto craneal, que puede considerarse parte del espectro clínico de AOS cuando se acompaña de defectos terminales transversos de extremidades.

Por los hallazgos de la hija anterior, se había considerado el diagnóstico clínico de síndrome de bandas amnióticas. Este síndrome es un defecto común, que generalmente presenta defectos de reducción de miembros. Streeter ${ }^{15}$ sugiere, en su teoría intrínseca, un origen común de las malformaciones fetales y la formación de bandas fibrosas durante etapas embrionarias tempranas por alteración de discos germinales, por lo que la presencia de bandas amnióticas como la detectada en nuestro propósito podría formar parte de una misma alteración. Esto se apoya también con la presencia de anillos de constricción reportados en diversos pacientes con AOS. ${ }^{6}$

Es por esto por lo que se sugiere un estudio detallado de la placenta en todos los pacientes con anillos de constricción y defecto terminal transversal de extremidades.

El diagnóstico de nuestra paciente se realizó sobre la base de la presencia de aplasia cutis congénita y defecto terminal transverso de extremidades. Sus extremidades inferiores, al igual que en el $78 \%$ de pacientes con AOS, no presentaban anomalías. ${ }^{14}$ Sin embargo, en nuestra paciente, se encontró labio y paladar hendido, una anomalía rara en esta enfermedad. ${ }^{14}$

Los estudios moleculares de AOS han mostrado heterogeneidad genética. Las mutaciones en ARHGAP31 y RBPJ pueden ser responsables del fenotipo grave observado en nuestros pacientes. El modelo de herencia autosómico recesivo no se consideró por tratarse de dos hijos afectados de padres diferentes. El mosaicismo gonadal materno no puede descartarse en nuestro caso. Las muestras de esta familia fueron amablemente aceptadas por el Dr. Trembath para su análisis molecular. El análisis de mutaciones de ganancia de función del gen ARHGAP31 resultó negativo y se continúa el estudio de genes candidatos. Sería interesante continuar la investigación de otros genes de la vía de señalización involucrada en AOS para entender la fisiopatología y heterogeneidad clínica de esta enfermedad.

\section{Agradecimientos}

Al Dr. Richard C. Trembath y a su equipo del Departamento de Genética Médica y Molecular de la Real Escuela de Medicina por recibir las muestras de las familias para el análisis molecular de AOS. 


\section{BIBLIOGRAFÍA}

1. Adams FH, Oliver CP. Hereditary deformities in man due to arrested development. J Hered 1945;36:3-7.

2. Küster W, Lenz W, Kääriäinen H, Majewski F. Congenital scalp defects with distal limb anomalies (Adams-Oliver syndrome): report of ten cases and review of the literature. Am J Med Genet 1988;31(1):99-115.

3. Chitayat D, Meunier C, Hodgkinson K, Robb L, Azouz M. Acrania: A Manifestation of the Adams-Oliver Syndrome. Am J Med Genet 1992;44(5):562-6.

4. Fryns JP.Congenital scalp defects with distal limb reduction anomalies. J Med Genet 1987;24(8):493-6.

5. Temtamy SA, Aglan MS, Ashour AM, Zaki MS. AdamsOliver syndrome: Further evidence of an autosomal recessive variant. Clin Dysmorphol 2007;16(3):141-9.

6. Savarirayan R, Thompson EM, Abbott KJ, Moore MH. Cerebralcortical dysplasia and digital constriction rings in Adams-Oliver syndrome. Am J Med Genet 1999;86(1):15-9.

7. Southgate L, Machado R, Snape K, Primeau M, et al. Gainof-Function Mutations of ARHGAP31, a Cdc42/Rac1 GTPase Regulator, cause Syndromic Cutis Aplasia and Limb Anomalies. Am J Hum Genet 2011;88(5):574-85.

8. Hoyme HE, Jones KL, Van Allen MI, Saunders BS, Benirschke K. Vascular pathogenesis of transverse limb reduction defects. J Pediatr 1982;101(5):839-43.
9. Hassed SJ, Wiley G, Wang S, Lee J, et al. RBPJ Mutations Identified in Two Families Affected by Adams-Oliver Syndrome. Am J Hum Genet 2012;91(2):391-5.

10. Vauclair S, Nicolas M, Barrandon Y, Radtke F. Notch1 is essential for postnatal hair follicle development and homeostasis. Dev Biol 2005;284(1):184-93.

11. Dou GR, Wang Y,Hu X, Hu L, et al. RBP-J, the transcription factor downstream of Notch receptors, is essential for the maintenance of vascular homeostasis in adult mice. FASEB I 2008;22(5):1606-17.

12. Shaheen R, Fageih E, Sunker A, Morsy H, et al. Recessive Mutations in DOCK6, Encoding the Guanidine Nucleotide Exchange Factor DOCK6, Lead to Abnormal Actin Cytoskeleton Organization and Adams-Oliver Syndrome. Am J Hum Genet 2011;89(2):328-33.

13. Shaheen R, Aglan M, Keppler-Noreuil K, Fageih E, et al. Mutations in EOGT confirm the genetic heterogeneity of autosomal-recessive Adams Oliver syndrome. Am J Hum Genet 2013;92(4): 598-604.

14. Farrell SA, Warda LJ, LaFlair P, Szymonowicz. AdamsOliver syndrome: A case with juvenile chronic myelogenous leukemia and chylothorax. Am J Med Genet 1993;47(8):1175-9.

15. Streeter GL. Focal deficiencies in fetal tissues and their relation to intrauterine amputation. Contrib Embryol Carnegie Inst 1930;22:1-44. 